\title{
LINEAR PROJECTIONS AND SUCCESSIVE MINIMA
}

\author{
CHRISTOPHE SOULÉ
}

\begin{abstract}
Let $X$ be an arithmetic surface, and let $L$ be a line bundle on $X$. Choose a metric $h$ on the lattice $\Lambda$ of sections of $L$ over $X$. When the degree of the generic fiber of $X$ is large enough, we get lower bounds for the successive minima of $(\Lambda, h)$ in terms of the normalized height of $X$. The proof uses an effective version (due to C. Voisin) of a theorem of Segre on linear projections and Morrison's proof that smooth projective curves of high degree are Chow semistable.
\end{abstract}

In arithmetic geometry, cohomology groups are not vector spaces as in classical algebraic geometry but rather are Euclidean lattices. As a consequence, to understand these groups we need to evaluate not only their rank but also their successive minima, which are fundamental invariants in the geometry of numbers. The goal of this article is to perform this task for line bundles on projective curves.

Let $K$ be a number field, let $\mathcal{O}_{K}$ be its ring of integers, and let $E$ be a projective $\mathcal{O}_{K}$-module of finite rank $N$. We endow $E \otimes_{\mathbb{Z}} \mathbb{C}$ with a Hermitian metric $h$, and we let $\mu_{1}, \ldots, \mu_{N}$ be the logarithm of the successive minima of $(E, h)$. Assume that $X_{K} \subset \mathbb{P}\left(E_{K}^{\vee}\right)$ is a smooth geometrically irreducible curve of genus $g>0$. We shall find a lower bound for the numbers $\mu_{i}$, $g+8 \leq i \leq N-3$, in terms of a normalized height of $X_{K}$ and the average of the $\mu_{i}$ (Theorem 2). This result is a complement to [13, Theorem 4], which gives a lower bound for $\mu_{1}$.

The method of proof is a variant of [13, Theorem 4]. It relies upon Morrison's [11] proof of the fact that $X_{K}$ is Chow semistable. We use a filtration $V_{1}=E_{K} \supset V_{2} \supset \cdots \supset V_{N}$ of the vector space $E_{K}$. But, contrary to [13], this filtration is chosen such that, for suitable values of $i$, the projection $\mathbb{P}\left(V_{i}^{\vee}\right) \cdots \rightarrow \mathbb{P}\left(V_{i+1}^{\vee}\right)$ does not change the degree of the image of $X_{K}$. That such a choice is possible follows from a result of Voisin, namely, an effective version of a theorem of Segre on linear projections of complex projective

Received November 15, 2008. Accepted July 28, 2009.

2000 Mathematics Subject Classification. 14H99, 14G40, 11 H06.

(C) 2010 by The Editorial Board of the Nagoya Mathematical Journal 
curves (Theorem 1). I thank C. Voisin for proving that result and for helpful discussions. I am also grateful to the referee for useful corrections.

\section{$\S 1$. Linear projections of projective curves}

Let $C \subset \mathbb{P}^{n}$ be an integral projective curve over $\mathbb{C}$, and let $d$ be its degree. Assume that $C$ is not contained in some hyperplane, that $d \geq 3$, and that $n \geq 3$.

TheOREM 1 (Voisin). There exists an integer $A(d)$ and a finite set $\Sigma$ of points in $\left(\mathbb{P}^{n}-C\right)(\mathbb{C})$, of order at most $A(d)$, such that, for every point $P \in \mathbb{P}^{n}(\mathbb{C})-\Sigma \cup C(\mathbb{C})$, the linear projection $\mathbb{P}^{n} \cdots \rightarrow \mathbb{P}^{n-1}$ of center $P$ maps $C$ birationally onto its image.

Proof. The existence of a finite set $\Sigma$ with the property above is a special case of a theorem of Segre (see [5]). The order of $\Sigma$ can be bounded as follows by a function of $d$.

If $n>3$, a generic linear projection into $\mathbb{P}^{3}$ will map $C$ isomorphically onto its image [10], and the exceptional set $\Sigma \subset \mathbb{P}^{n}$ will map bijectively onto the exceptional set in $\mathbb{P}^{3}$. Therefore we can assume that $n=3$.

When the projection with center $P \in \mathbb{P}^{3}(\mathbb{C})$ is not birational from the curve $C$ to its image $C^{\prime} \subset \mathbb{P}^{2}$, we have $d^{\prime}=\operatorname{deg}\left(C^{\prime}\right) \leq d / 2$; hence $d^{\prime} \leq d-2$, and $P$ is the vertex of a cone $K$ with base $C^{\prime}$ containing $C$. So we have to bound the number of such cones.

Let $N$ be the dimension of the kernel of the restriction map

$$
\alpha: H^{0}\left(\mathbb{P}^{3}, \mathcal{O}\left(d^{\prime}\right)\right) \rightarrow H^{0}\left(C, \mathcal{O}\left(d^{\prime}\right)\right)
$$

Clearly, $N$ is bounded as a function of $d$, and any $f \in \operatorname{ker}(\alpha)$ is a homogeneous polynomial of degree $d^{\prime}$ that vanishes on $C$.

Let $Z \subset \mathbb{P}^{3}(\mathbb{C}) \times \mathbb{P}^{N-1}(\mathbb{C})$ be the set of pairs $(P, f)$ such that $f$ is the equation of a cone $K$ of vertex $P$. If $p_{1}: \mathbb{P}^{3} \times \mathbb{P}^{N-1} \rightarrow \mathbb{P}^{3}$ is the first projection, we have to bound the order of $p_{1}(Z)$. We note that this order is at most the number $c$ of connected components of $Z$.

Now $Z$ is defined by equations of bidegree $(\delta, 1), \delta \leq d^{\prime}$. Indeed, $f$ is homogeneous of degree $d^{\prime}$, and $(P, f) \in Z$ when all the derivatives of $f$, except those of order $d^{\prime}$, vanish at $P$.

Let $L=\mathcal{O}\left(d^{\prime}, 1\right)$, let $M=\operatorname{dim} H^{0}\left(\mathbb{P}^{3} \times \mathbb{P}^{N}, L\right)-1$, and let

$$
j: \mathbb{P}^{3} \times \mathbb{P}^{N} \rightarrow \mathbb{P}^{M}
$$


be the Segre embedding. Since $j(Z)$ is the intersection of $j\left(\mathbb{P}^{3} \times \mathbb{P}^{N}\right)$ with linear hyperplanes, the Bézout theorem [7, §8.4] tells us that

$$
c \leq \operatorname{deg}\left(j\left(\mathbb{P}^{3} \times \mathbb{P}^{N}\right)\right) .
$$

Hence $c$ is bounded by a function of $d$.

Corollary 1. Given any projective line $\Lambda \subset \mathbb{P}^{n}$, there exists a finite set $\Phi$ of order at most $A(d)+d$ in $\Lambda$ such that, if $P \in \Lambda-\Phi$, the linear projection of center $P$ maps $C$ birationally onto its image.

Proof. Since $C$ is not equal to $\Lambda$, the cardinality of $C \cap \Lambda$ is at most $d$. So the corollary follows from Theorem 1.

REMARK. The proof of Theorem 1 provides an upper bound for $A(d)$. Indeed,

$$
\operatorname{deg}(j)=\left(\begin{array}{c}
3+N \\
3
\end{array}\right) d^{\prime 3},
$$

$d^{\prime} \leq d / 2$, and

$$
N=\operatorname{dim} H^{0}\left(\mathbb{P}^{3}, \mathcal{O}\left(d^{\prime}\right)\right)=\frac{d^{\prime 3}}{6}+d^{\prime 2}+\frac{11}{6} d^{\prime}+1
$$

In particular, when $d \geq 12$, we get

$$
\log (A(d)+d) \leq 12 \log (d)-12 .
$$

\section{$\S 2$. Successive minima}

\section{1 .}

Let $K$ be a number field, let $[K: \mathbb{Q}]$ be its degree over $\mathbb{Q}$, let $\mathcal{O}_{K}$ be its ring of integers, let $S=\operatorname{Spec}\left(\mathcal{O}_{K}\right)$ be the associated scheme, and let $\Sigma$ be the set of complex embeddings of $K$. Consider a Hermitian vector bundle $(E, h)$ over $S$; that is, $E$ is a torsion-free $\mathcal{O}_{K}$-module of finite rank $N$, and for all $\sigma \in \Sigma$, the associated complex vector space $E_{\sigma}=E \otimes \mathcal{O}_{K} \mathbb{C}$ is equipped with a Hermitian scalar product $h_{\sigma}$. If $\bar{\sigma}$ is the conjugate of $\sigma$, we assume that the complex conjugation $E_{\sigma} \simeq E_{\bar{\sigma}}$ is an isometry. If $v \in E$, we define

$$
\|v\|=\max _{\sigma \in \Sigma} \sqrt{h_{\sigma}(v, v)}
$$


If $i$ is a positive integer, $i \leq N$, we let $\mu_{i}$ be the infimum of the set of real numbers $r$ such that there exist $v_{1}, \ldots, v_{i} \in E$, linearly independent over $K$, such that $\log \left\|v_{\alpha}\right\| \leq r$ for all $\alpha \leq i$. The number $\mu_{i}$ is thus the logarithm of the $i$ th successive minimum of $(E, h)$. Let

$$
\mu=\frac{\mu_{1}+\cdots+\mu_{N}}{N} .
$$

\section{2 .}

If $E^{\vee}=\operatorname{Hom}\left(E, \mathcal{O}_{K}\right)$ is the dual of $E$, we let $\mathbb{P}\left(E^{\vee}\right)$ be the associated projective space, representing lines in $E^{\vee}$. Let $E_{K}^{\vee}=E^{\vee} \otimes_{\mathcal{O}_{K}} K$, and let $X_{K} \subset \mathbb{P}\left(E_{K}^{\vee}\right)$ be a smooth geometrically irreducible curve of genus $g$ and degree $d$. We assume that the embedding of $X_{K}$ into $\mathbb{P}\left(E_{K}^{\vee}\right)$ is defined by a complete linear series on $X_{K}$. We also assume that $d \geq 2 g+1$. The rank of $E$ is thus $N=d+1-g$.

If $X$ is the Zariski closure of $X_{K}$ in $\mathbb{P}\left(E^{\vee}\right)$, and if $\overline{\mathcal{O}(1)}$ is the canonical Hermitian line bundle on $\mathbb{P}\left(E^{\vee}\right)$, the Faltings height of $X_{K}$ is the real number

$$
h\left(X_{K}\right)=\widehat{\operatorname{deg}}\left(\hat{c}_{1}(\overline{\mathcal{O}(1)})^{2} \mid X\right)
$$

(see $[2,(3.1 .1)$ and $(3.1 .5)])$.

\section{3.}

For any positive integer $i \leq N$, we define the integer $f_{i}$ by the formulas

$$
f_{i}=i-1 \quad \text { if } i-1 \leq d-2 g
$$

and

$$
f_{i}=i-1+\alpha \quad \text { if } i-1=d-2 g+\alpha, \quad 0 \leq \alpha \leq g .
$$

Assume that $k$ and $i$ are two positive integers, $k \leq N, i \leq N$. We let

$$
h_{i, k}= \begin{cases}f_{i} & \text { if } i \leq k, i=N-1, \text { or } i=N, \\ f_{k} & \text { if } k \leq i \leq N-2 .\end{cases}
$$

Finally, if $2 \leq k \leq N$, we let

$$
B_{k}=\max _{i=2, \ldots, N} \frac{h_{i, k}^{2}}{(i-1) h_{i, k}-\sum_{j=1}^{i-1} h_{j, k}} .
$$

Proposition 1. For every $k$ such that $2 \leq k \leq N-3$, the following inequality holds:

$B_{k}\left(\mu_{N+1-k}-\mu_{1}\right)+\frac{h\left(X_{K}\right)}{[K: \mathbb{Q}]}+2 d \mu \geq\left(2 d-N B_{k}\right)\left(\mu-\mu_{1}\right)-2 d \log (A(d)+d)$. 


\section{4.}

From Proposition 1, we shall deduce the following result.

THEOREM 2. If $g>0$, for every integer $k$ such that $4 \leq k \leq d-2 g-6$, the following inequality holds:

$$
\mu_{N+1-k}-\mu \geq-k\left(\frac{h\left(X_{K}\right)}{2 d[K: \mathbb{Q}]}+\mu\right)-12 d \log (d)+12 d .
$$

REMARKS. (i) It is proved in [13] that, when $d \geq 2 g+1$,

$$
\frac{h\left(X_{K}\right)}{2 d[K: \mathbb{Q}]}+\mu \geq \frac{2 g(d-2 g)}{d^{2}+d-2 g^{2}}\left(\mu-\mu_{1}\right) .
$$

In particular,

$$
\frac{h\left(X_{K}\right)}{2 d[K: \mathbb{Q}]}+\mu \geq 0 .
$$

(ii) From Bombieri and Vaaler's version of Minkowski's theorem on successive minima (see [4]), we know that

$$
-\mu \geq \frac{\widehat{\operatorname{deg}}(E, h)}{N[K: \mathbb{Q}]}-\frac{\log \left|D_{K}\right|}{2[K: \mathbb{Q}]}-K(N),
$$

where $D_{K}$ is the absolute discriminant of $K$ and the constant $K(N)$ depends only on $N$. If $h_{L^{2}}$ is the $L^{2}$-metric on $H^{0}(X, \mathcal{O}(1))$ (see [3, (1.2.3)]), the quantity

$$
\frac{1}{[K: \mathbb{Q}]}\left(\frac{h\left(X_{K}\right)}{2 d}-\frac{\widehat{\operatorname{deg}}\left(H^{0}(X, \mathcal{O}(1)), h_{L^{2}}\right)}{N}\right)
$$

is the normalized height of $X_{K}$ introduced by Bost [3, (1.2.4)].

\section{5.}

To prove Proposition 1, fix a positive integer $k \leq N-3$ and choose elements $x_{1}, \ldots, x_{N}$ in $E$, linearly independent over $K$ and such that

$$
\log \left\|x_{i}\right\|=\mu_{N-i+1}, \quad 1 \leq i \leq N .
$$

Fix integers $n_{\alpha}, \alpha=k+1, \ldots, N-2$, to be specified later (in $\S 2.7$ ). If $1 \leq i \leq N$, we define

$$
v_{i}= \begin{cases}x_{i}+n_{i} x_{i-1} & \text { if } k+1 \leq i \leq N-2 \\ x_{i} & \text { otherwise }\end{cases}
$$


We get a complete flag $E_{K}=V_{1} \supset V_{2} \supset \cdots \supset V_{N}$ by defining $V_{i}$ to be the linear span of $v_{i}, v_{i+1}, \ldots, v_{N}$.

When $m$ is large enough, the cup-product map

$$
\varphi: E_{K}^{\otimes m} \rightarrow H^{0}\left(X_{K}, \mathcal{O}(m)\right)
$$

is surjective; hence $H^{0}\left(X_{K}, \mathcal{O}(m)\right)$ is generated by the monomials

$$
v_{1}^{\alpha_{1}} \cdots v_{N}^{\alpha_{N}}=\varphi\left(v_{1}^{\otimes \alpha_{1}} \cdots v_{N}^{\otimes \alpha_{N}}\right),
$$

$\alpha_{1}+\cdots+\alpha_{N}=m$. A special basis of $H^{0}\left(X_{K}, \mathcal{O}(m)\right)$ is a basis made of such monomials.

Let $r_{1}, r_{2}, \ldots, r_{N}$ be $N$ real numbers, and let $\boldsymbol{r}=\left(r_{1}, \ldots, r_{N}\right)$. We define the weight of $v_{i}$ to be $r_{i}$, we define the weight of a monomial in $E_{K}^{\otimes m}$ to be the sum of the weights of the $v_{i}$ occurring in it, and we define the weight of a monomial $u \in H^{0}\left(X_{K}, \mathcal{O}(m)\right)$ to be the minimum $w t_{\boldsymbol{r}}(u)$ of the weights of the monomials in the $v_{i}$ mapping to $u$ by $\varphi$. The weight $w t_{\boldsymbol{r}}(\mathcal{B})$ of a special basis $\mathcal{B}$ is the sum of the weights of its elements, and $w_{\boldsymbol{r}}(m)$ is the minimum of the weights of special bases of $H^{0}\left(X_{K}, \mathcal{O}(m)\right)$.

When $r_{1} \geq r_{2} \geq \cdots \geq r_{N}=0$ are natural integers, there exists $e_{\boldsymbol{r}} \in \mathbb{N}$ such that, as $m$ goes to infinity,

$$
w_{\boldsymbol{r}}(m)=e_{\boldsymbol{r}} \frac{m^{2}}{2}+O(m)
$$

(see [12], [11, Corollary 3.3]).

Our next goal is to find an upper bound for $e_{\boldsymbol{r}}$.

\section{6.}

For every positive integer $i \leq N$, we let $e_{i}$ be the drop in degree of $X_{K}$ when projected from $\mathbb{P}\left(E_{K}^{\vee}\right)$ to $\mathbb{P}\left(V_{i}^{\vee}\right)$. A criterion of Gieseker (see [8], [11, Corollary 3.8]) tells us that $e_{\boldsymbol{r}} \leq S$ with

$$
S=\min _{1=i_{0}<\cdots<i_{\ell}=N} \sum_{j=0}^{\ell-1}\left(r_{i_{j}}-r_{i_{j+1}}\right)\left(e_{i_{j}}+e_{i_{j+1}}\right) .
$$

Note that $S$ is an increasing function in each variable $e_{i}$. Furthermore, it follows from Clifford's theorem and Riemann-Roch that

$$
e_{i} \leq f_{i}
$$

for every positive $i \leq N$ (see [11, proof of Theorem 4.4]). (Note that in [11, Theorem 4.4], the filtration of $V_{0}$ has length $n+1$, while $n=\operatorname{dim} V_{0}$. In our case, we start the filtration with $V_{1}$, hence the discrepancy between our definition of $f_{i}$ and that of [11].) 


\section{7.}

We now specify our choice of the integers $n_{i}$ in (2). This is where our argument will differ from that of [13, Theorem 4], which corresponds to the choice $n_{i}=0$ for every $i$.

Let $w_{1}, \ldots, w_{N} \in E_{K}^{\vee}$ be the dual basis of $v_{1}, \ldots, v_{N}$. The linear projection from $\mathbb{P}\left(V_{i}^{\vee}\right)$ to $\mathbb{P}\left(V_{i+1}^{\vee}\right)$ has center the image $\dot{w}_{i}$ of $w_{i}$.

If $y_{1}, \ldots, y_{N} \in E_{K}^{\vee}$ is the dual basis of $x_{1}, \ldots, x_{N}$, then we get

$$
w_{i}= \begin{cases}y_{i}+n_{i} z_{i} & \text { if } k \leq i \leq N-3 \\ y_{i} & \text { otherwise }\end{cases}
$$

where $z_{i}+y_{i+1}$ is a linear combination of $y_{i+2}, y_{i+3}, \ldots$ with coefficients depending only on $n_{i+1}, n_{i+2}, \ldots$ When $n \neq m$ are two integers, the vectors $y_{i}+n z_{i}$ and $y_{i}+m z_{i}$ are linearly independent over $K$; therefore, their images in $\mathbb{P}\left(V_{i}^{\vee}\right)$ are distinct. Since $e_{N-3} \leq f_{N-3}$ and $g>0$, we get $e_{N-3} \leq d-3$; therefore, the image of $X_{K}$ in $\mathbb{P}\left(V_{i}^{\vee}\right), i \leq N-3$, has degree at least 3 . Furthermore, $\operatorname{dim} \mathbb{P}\left(V_{i}^{\vee}\right) \geq 3$. By Theorem 1 and Corollary 1, it follows that we can choose $n_{i}$ such that $0 \leq n_{i}<A(d)+d$ and the projection from $\mathbb{P}\left(V_{i}^{\vee}\right)$ to $\mathbb{P}\left(V_{i+1}^{\vee}\right)$ does not change the degree of the image of $X_{K}$. We fix the integers $n_{i}, k \leq i \leq N-3$, with this property. Hence we have

$$
e_{i}=e_{k} \quad \text { whenever } k \leq i \leq N-2 \text {. }
$$

\section{8 .}

From (3) and (4), we conclude that

$$
e_{i} \leq h_{i, k} \quad \text { if } 1 \leq i \leq N
$$

(see §2.3). Hence, by Morrison's main combinatorial theorem [11, Corollary 4.3], for any decreasing sequence of real numbers $r_{1} \geq r_{2} \geq \cdots \geq r_{N}$ we have, if $k \geq 2$,

$$
S \leq \psi(\boldsymbol{r})
$$

with

$$
\psi(\boldsymbol{r})=B_{k} \cdot \sum_{i=1}^{N}\left(r_{i}-r_{N}\right) .
$$

So, when $r_{1} \geq r_{2} \geq \cdots \geq r_{N}=0$ is a decreasing sequence of integers,

$$
e_{\boldsymbol{r}} \leq \psi(\boldsymbol{r})
$$


Let

$$
s_{i}= \begin{cases}\log \left\|x_{i-1}\right\|+\log (A+d) & \text { if } k+1 \leq i \leq N-2, \\ \log \left\|x_{i}\right\|+\log (A+d) & \text { otherwise }\end{cases}
$$

and let $r_{i}=s_{i}-s_{N}$. The sequence $r_{1}, \ldots, r_{N}=0$ is decreasing, and (2) implies that

$$
\log \left\|v_{i}\right\| \leq \log \left\|x_{i-1}\right\|+\log \left(1+n_{i}\right) \leq s_{i} \quad \text { if } k+1 \leq i \leq N-2,
$$

and

$$
\log \left\|v_{i}\right\|=\log \left\|x_{i}\right\| \leq s_{i} \quad \text { otherwise. }
$$

We endow $\mathcal{O}(1)$ with the metric induced by the metric $h$ on $E$. We choose a Hermitian metric, invariant by complex conjugation, on the complex points of $X$, and we endow $M=H^{0}(X, \mathcal{O}(m))$ with the associated $L^{2}$-metric. After multiplying the metric on $X$ by a fixed constant, we know that, for every $m$, the morphism $\varphi$ is norm decreasing. Therefore, if $u=$ $\varphi\left(v_{1}^{\otimes \alpha_{1}} \cdots v_{N}^{\otimes \alpha_{N}}\right)$ is a monomial in $M$, we have

$$
\log \|u\| \leq \sum_{i=1}^{N} \alpha_{i} \log \left\|v_{i}\right\| \leq \sum_{i=1}^{N} \alpha_{i} s_{i}=\sum_{i=1}^{N} \alpha_{i} r_{i}+m s_{N}
$$

By definition of $w t_{r}(u)$, we get

$$
\log \|u\| \leq w t_{\boldsymbol{r}}(u)+m s_{N} .
$$

Let $\widehat{\operatorname{deg}}(\bar{M})$ be the arithmetic degree of the Hermitian vector bundle $\bar{M}=$ $\left(M, h_{L^{2}}\right)$ over $S$. From the inequality above and the Hadamard inequality, we deduce that, for any special basis $\mathcal{B}$ of $M$,

$$
\widehat{\operatorname{deg}}(\bar{M}) \geq-[K: \mathbb{Q}] \sum_{u \in \mathcal{B}}\left(w t_{\boldsymbol{r}}(u)+m s_{N}\right) .
$$

This implies that

$$
\widehat{\operatorname{deg}}(\bar{M}) \geq-[K: \mathbb{Q}]\left(w_{\boldsymbol{r}}(m)+m h^{0}\left(X_{K}, \mathcal{O}(m)\right) s_{N}\right) .
$$

By (5) and the definition of $e_{\boldsymbol{r}}$, since both $w_{\boldsymbol{r}}(m)$ and $\psi(\boldsymbol{r})$ are linear functions of $\boldsymbol{r}$, by approximating $\boldsymbol{r}$ by a collection of rational numbers we get that, for every positive real number $\eta$,

$$
w_{\boldsymbol{r}}(m) \leq(\psi(\boldsymbol{r})+\eta) \frac{m^{2}}{2}+O(m)
$$


(see $[13, \S 2.2])$, so we get

$$
\widehat{\operatorname{deg}}(\bar{M}) \geq-[K: \mathbb{Q}]\left(\psi(\boldsymbol{r})+2 d s_{N}+\eta\right) \frac{m^{2}}{2}+O(m) .
$$

On the other hand, by [6, Corollary to Theorem 3] and [9, Theorem 8], we have

$$
\widehat{\operatorname{deg}}(\bar{M})=h\left(X_{K}\right) \frac{m^{2}}{2}+O(m \log (m))
$$

Therefore, for every $\eta>0$,

$$
h\left(X_{K}\right) \geq-[K: \mathbb{Q}]\left(\psi(\boldsymbol{r})+2 d s_{N}+\eta\right) .
$$

By the definition of $\psi$, we deduce from (7) that

$$
\frac{h\left(X_{K}\right)}{[K: \mathbb{Q}]}+2 d s_{N} \geq-B_{k}\left(\sum_{i=1}^{N} r_{i}\right)
$$

and, using (6), that

(8) $\frac{h\left(X_{K}\right)}{[K: \mathbb{Q}]}+2 d \mu_{1} \geq-B_{k}\left(\sum_{i=1}^{N}\left(\mu_{i}-\mu_{1}\right)+\mu_{N+1-k}-\mu_{3}\right)-2 d \log (A(d)+d)$.

Since $\mu_{3} \geq \mu_{1}$, (8) implies the inequality in Proposition 1 .

2.9 .

To make Proposition 1 more explicit, we need to evaluate $B_{k}$. For any $i=2, \ldots, N$, we let

$$
B_{i, k}=\frac{h_{i, k}^{2}}{(i-1) h_{i, k}-\sum_{j=1}^{i-1} h_{j, k}}
$$

so that

$$
B_{k}=\sup _{i} B_{i, k}
$$

Lemma 1. Assume that $k-1 \leq d-2 g$. Then

- if $i \leq k$,

$$
B_{i, k}=2-\frac{2}{i}
$$

- if $k \leq i \leq N-2$,

$$
B_{i, k}=2-\frac{2}{k}
$$




\subsection{0.}

To prove Lemma 1 , we first assume that $i \leq k$. Then, if $j \leq i$, we have

$$
h_{j, k}=f_{j}=j-1 \text {. }
$$

Therefore,

$$
\sum_{j=1}^{i-1} h_{j, k}=\sum_{j=1}^{i-1}(j-1)=\frac{(i-2)(i-1)}{2}
$$

and

$$
B_{i, k}=\frac{(i-1)^{2}}{(i-1)^{2}-\frac{(i-2)(i-1)}{2}}=2-\frac{2}{i} .
$$

Assume now that $k \leq i \leq N-2$. Then, if $1 \leq j \leq k-1$, we have

$$
h_{j, k}=f_{j}=j-1 .
$$

Furthermore, if $k \leq j \leq i-1$, we get

$$
h_{j, k}=f_{k}=k-1 \text {. }
$$

Therefore,

$$
B_{i, k}=\frac{(k-1)^{2}}{(i-1)(k-1)-\sum_{j=1}^{k-1}(j-1)-\sum_{j=k}^{i-1}(k-1)}=2-\frac{2}{k} .
$$

\subsection{1 .}

Lemma 2. Assume that $g>0$ and that $4 \leq k \leq d-2 g-6$. Then $B_{k}<2$ and

$$
2 d-N B_{k} \geq \frac{2 d}{k}
$$

To prove Lemma 2, we first compute $B_{N-1, k}$ and $B_{N, k}$ under the assumption $k-1 \leq d-2 g$. When $d-2 g \leq i-1<N$, we have

$$
f_{i}=2 i-2-d+2 g
$$

Therefore,

$$
h_{N-1, k}=f_{N-1}=2(N-2)-d+2 g=d-2,
$$

and

$$
h_{N, k}=f_{N}=2(N-1)-d+2 g=d .
$$


On the other hand,

$$
\begin{aligned}
\sum_{j=1}^{N-2} h_{j, k} & =\sum_{j=1}^{k} f_{j}+\sum_{j=k+1}^{N-2} f_{k} \\
& =\sum_{j=1}^{k}(j-1)+\sum_{j=k+1}^{N-2}(k-1),
\end{aligned}
$$

and

$$
\sum_{j=1}^{N-1} h_{j, k}=\sum_{j=1}^{N-2} h_{j, k}+f_{N-1}
$$

Therefore,

$$
B_{N-1, k}=\frac{(d-2)^{2}}{(N-2)(d-2)-\sum_{j=1}^{k}(j-1)-\sum_{j=k+1}^{N-2}(k-1)}
$$

$$
=\frac{(d-2)^{2}}{(N-2)(d-1-k)+\frac{k(k-1)}{2}},
$$

and

$$
\begin{aligned}
B_{N, k} & =\frac{d^{2}}{(N-2) d+2-\sum_{j=1}^{k}(j-1)-\sum_{j=k+1}^{N-2}(k-1)} \\
& =\frac{d^{2}}{2+(N-2)(d+1-k)+\frac{k(k-1)}{2}}
\end{aligned}
$$

When $i \leq N-2$, we know from Lemma 1 that

$$
B_{i, k} \leq 2-\frac{2}{k}<2
$$

hence,

$$
2 d-N B_{i, k} \geq 2 d-(d+1-g)\left(2-\frac{2}{k}\right) \geq \frac{2 d}{k} .
$$

When $i=N-1$, we put $k=4+p, p \geq 0$, and $d=10+2 g+p+t, t \geq 0$. Then (9) implies that $2-B_{N-1, k}$ has the same sign as $2 g t+14 g+p+t^{2}+$ $12 t+38$, which is positive. On the other hand,

$$
2 d-N B_{N-1, k}-\frac{2 d}{k}
$$


has the sign of the numerator of $k-1-N k B_{N-1, k} /(2 d)$, namely,

$$
\begin{aligned}
(2+ & p) t^{3}+\left(p^{2}+(5 g+21) p+10 g+36\right) t^{2} \\
& +\left((8+4 g) p^{2}+\left(8 g^{2}+121+72 g\right) p+186+118 g+16 g^{2}\right) t+(g-1) p^{3} \\
& +\left(5+26 g+4 g^{2}\right) p^{2}+\left(172+4 g^{3}+60 g^{2}+240 g\right) p \\
& +244+328 g+92 g^{2}+8 g^{3}>0 .
\end{aligned}
$$

Similarly, (10) implies that $2-B_{N, k}$ has the same sign as $t^{2}+(2 g+12) t+$ $42+10 g+p$, so it is positive, and

$$
2 d-N B_{N, k}-\frac{2 d}{k}
$$

has the sign of

$$
\begin{aligned}
& (2+p) t^{2}+((9+3 g) p+12+6 g) t+(g-1) p^{2}+\left(11+18 g+2 g^{2}\right) p \\
& -14+22 g+4 g^{2}>0 .
\end{aligned}
$$

This ends the proof of Lemma 2 .

\subsection{2.}

To prove Theorem 2 , we first note that $B_{k}<2$ and $2 d-N B_{k} \geq 2 d / k$ by Lemma 2. Since $\mu_{1} \leq \mu_{N+1-k}$ and $\mu_{1} \leq \mu$, Proposition 1 implies that

$$
2\left(\mu_{N+1-k}-\mu_{1}\right)+\frac{h\left(X_{K}\right)}{[K: \mathbb{Q}]}+2 d \mu \geq \frac{2 d}{k}\left(\mu-\mu_{1}\right)-2 d \log (A(d)+d) .
$$

By dividing this inequality by $2 d$, we obtain

$$
\frac{1}{d}\left(\mu_{N+1-k}-\mu_{1}\right)+\frac{h\left(X_{K}\right)}{2 d[K: \mathbb{Q}]}+\mu \geq \frac{1}{k}\left(\mu-\mu_{1}\right)-\log (A(d)+d) .
$$

Since $k<d$, we get

$$
\mu_{N+1-k}-\mu_{1}+k\left(\frac{h\left(X_{K}\right)}{2 d[K: \mathbb{Q}]}+\mu\right) \geq \mu-\mu_{1}-d \log (A(d)+d),
$$

and, since $d \geq 12$, the remark in $\S 1$ implies Theorem 2 . 


\section{REFERENCES}

[1] E. Arbarello, M. Cornalba, P. A. Griffiths, and J. Harris, Geometry of Algebraic Curves, I, Grundlehren Math. Wiss. 267, Springer, New York, 1985.

[2] J.-B. Bost, H. Gillet, and C. Soulé, Heights of projective varieties and positive Green forms, J. Am. Math. Soc., 7 (1994), 903-1027.

[3] J.-B. Bost, Intrinsic heights of stable varieties and abelian varieties, Duke Math. J., 82 (1996), 21-70.

[4] E. Bombieri and J. Vaaler, On Siegel's lemma, Invent. Math., 73 (1983), 11-32.

[5] A. Calabri and C. Ciliberto, On special projections of varieties: Epitome to a theorem of Beniamino Segre, Adv. Geom., 1 (2001), 97-106.

[6] G. Faltings, Calculus on arithmetic surfaces, Ann. of Math., 119 (1984), 387-424.

[7] W. Fulton, Intersection Theory, 2nd ed., Ergeb. Math. Grenzgeb. (3), 2, Springer, Berlin, 1998.

[8] D. Gieseker, Global moduli for surfaces of general type, Invent. Math., 43 (1977), 233-282.

[9] H. Gillet and C. Soulé, An arithmetic Riemann-Roch theorem, Invent. Math., 110 (1992), 473-543.

[10] R. Hartshorne, Algebraic Geometry, corr. 3rd printing, Grad. Texts Math. 52, Springer, New York, 1983.

[11] I. Morrison, Projective stability of ruled surfaces, Invent. Math., 56 (1980), 269-304.

[12] D. Mumford, Stability of projective varieties, Enseign. Math. (2), 23 (1977), 39-110.

[13] C. Soulé, Successive minima on arithmetic varieties, Compos. Math., 96 (1995), 85-98.

Institut des Hautes Études Scientifiques

35 Route de Chartres

91440 Bures-sur-Yvette

France

soule@ihes.fr 